\title{
What is new in severe sepsis in the critically ill patient?
}

Severe sepsis is common in critically ill patients and is responsible for high mortality and morbidity. The aim of this special issue is to highlight recent advances in epidemiology, pathophysiology, diagnosis and treatment of sepsis in the critically ill patient.

Dr. Nora and Dr. Povoa discuss the relationship between ventilator-associated pneumonia (VAP) rates and antibiotic consumption. It would be expected from the recent trends of reduction of VAP incidence that the consumption of antibiotics would also decrease, in particular in those hospitals with lowest VAP rates. However, ICU reports show a steadily use of antibiotics for nosocomial pneumonia. Knowledge of bacterial epidemiology and resistance profiles for each ICU has great relevance in order to understand trends of antibiotic use. Dr. Jaffal and colleagues report on the relationship between hyperoxemia and VAP. Hyperoxemia is responsible for denitrogenation phenomena, inhibition of surfactant production, promoting atelectasis in mechanically ventilated patients. Further, hyperoxemia impairs the efficacy of alveolar macrophages to migrate, phagocyte and kill bacteria. Oxygen can also cause pulmonary-specific toxic effect called hyperoxic acute lung injury leading to longer duration of mechanical ventilation. All these hyperoxic effects are well-known risk factors for VAP. Dr. Roux and colleagues discuss the adapted island theory that has been proposed for the colonization and infection of the respiratory tract. In this model, not only the immigration of bacteria into the lung is important, but elimination and regional growth factors are of equal significance. They suggest that there is a strong need for more in-depth analyses of the changes in the microbial composition of the pulmonary microbiome during mechanical ventilation and its relationship with the development of VAP.

Dr. Ryan and colleagues highlight the importance of immune compromise in the pathophysiology of sepsis and healthcareassociated infection. Septic patients appear to exhibit a complex and long-lasting immune deficiency state, involving lymphocytes of both the innate and adaptive immune responses, that have been linked with mortality and the occurrence of health care associated infection.

Point-of-care tools (POCT) are diagnostic testing methods that can be used at bedside, with delays ranging from a couple minutes to a few hours. Dr. Millot and colleagues discuss how the use of POCTs for VAP could allow faster diagnosis and antimicrobial therapy adjustments. Accuracy of multiplex polymerase chain reaction has been demonstrated, and clinical studies are ongoing to evaluate its interest at bedside in patients with pneumonia.

Inhaled antimicrobial treatment is an interesting option in ventilated patients suffering from VAP. Dr. de Pablo and colleagues discuss physicochemical parameters of optimal lung deposition and main characteristics of currently available nebulisers and formulations as well as novel nanotechnology approaches which are revolutionizing inhaled therapies especially in the treatment of lung cancer and infections. Dr. Juschten and colleagues report that in preclinical models, all tested anticoagulants were capable of attenuating pulmonary coagulopathy, but the effects on inflammation were less consistent. Nebulized heparin, reduced systemic coagulation, but human trials are inconclusive to draw firm conclusions regarding efficacy and safety. Based on pre-clinical studies, there is a growing evidence of the potential benefits of cell based therapies for the treatment of sepsis and ARDS. Dr. Guillamat-Prats and colleagues describe the different cell types, their potential sources and characteristics and mechanism of action. And also elucidate the results of several pre-clinical and clinical studies in ARDS and in sepsis and the future directions of these studies.

\section{Acknowledgements}

None. 


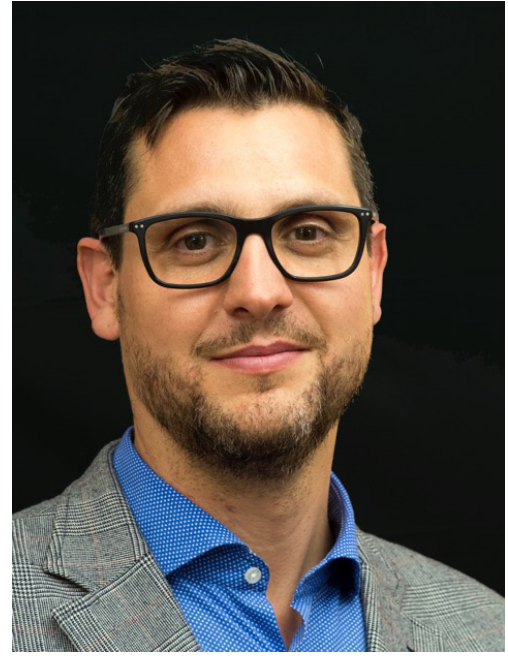

Ignacio Martin-Loeches

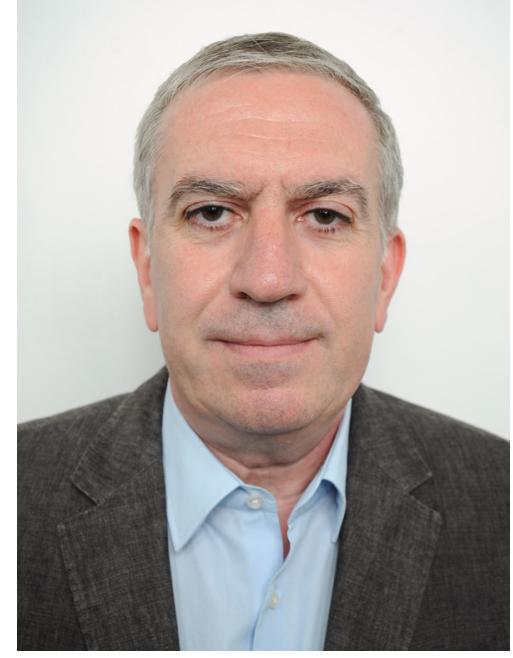

Saad Nseir

Ignacio Martin-Loeches ${ }^{1}$

${ }^{1}$ Multidisciplinary Intensive Care Research Organization (MICRO), Wellcome Trust-HRB Clinical Research, Department of Clinical Medicine, Trinity Centre for Health Sciences, St fames's University Hospital, Dublin, Ireland.

(Email:drmartinloeches@gmail.com)

Saad Nseir ${ }^{2,3}$

${ }^{2}$ Centre de Réanimation, CHU Lille, Lille, France; ${ }^{3}$ Lille University, Medicine School, Lille, France.

(Email:s-nseir@chru-lille.fr)

doi: $10.21037 / \mathrm{atm} .2017 .11 .27$

Conflicts of Interest: S Nseir: MSD, and Medtronic (lecture), CielMedical, and Bayer (advisory board);

I Martin-Loeches has no conflicts of interest to declare.

View this article at: http://dx.doi.org/10.21037/atm.2017.11.27

Cite this article as: Martin-Loeches I, Nseir S. What is new in severe sepsis in the critically ill patient? Ann Transl Med 2017;5(22):442. doi: 10.21037/atm.2017.11.27 